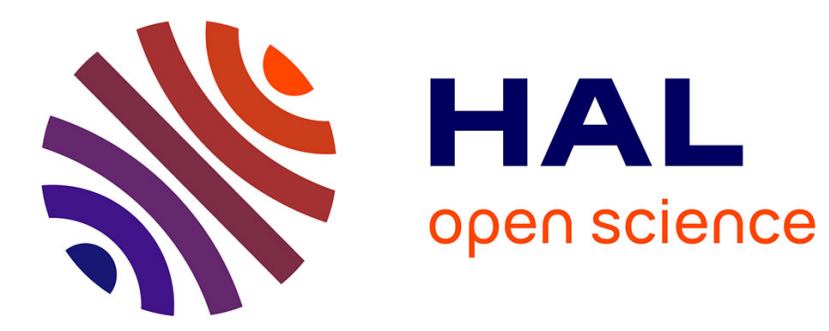

\title{
La professionnalisation des associations par les pratiques de gestion des compétences des acteurs associatifs produit-elle un processus de changement organisationnel?
}

Yolande François

\section{To cite this version:}

Yolande François. La professionnalisation des associations par les pratiques de gestion des compétences des acteurs associatifs produit-elle un processus de changement organisationnel?. Recherches en sciences de gestion, 2015, 106, pp.106. hal-01154940

\section{HAL Id: hal-01154940 \\ https://univ-lyon3.hal.science/hal-01154940}

Submitted on 25 May 2015

HAL is a multi-disciplinary open access archive for the deposit and dissemination of scientific research documents, whether they are published or not. The documents may come from teaching and research institutions in France or abroad, or from public or private research centers.
L'archive ouverte pluridisciplinaire HAL, est destinée au dépôt et à la diffusion de documents scientifiques de niveau recherche, publiés ou non, émanant des établissements d'enseignement et de recherche français ou étrangers, des laboratoires publics ou privés. 
revue Recherches en Sciences de Gestion-Management Sciences-Ciencias de Gestión, n²106, p.

\title{
La professionnalisation des associations par les pratiques de gestion des compétences des acteurs associatifs produit-elle un processus de changement organisationnel ?
}

\author{
Yolande François \\ Maître de conférences \\ ISEOR, Magellan \\ IAE Lyon, Université Jean Moulin
}

(France)

Cet article porte sur la professionnalisation des associations par l'identification des modes de gestion des compétences des acteurs associatifs. La question est de savoir la professionnalisation de l'association peut entraîner une modification de la structure associative vers un système pouvant être rapproché à une pratique privée d'organisation similaire. Ces associations arrivent- elles alors à atteindre ou non un certain degré d'organisation et de structuration jugé professionnel?

Mots-clés : Pratiques associatives, Gestion des compétences, Isomorphisme, changement.

This article focuses on the professionalization of associations by identifying ways of managing skills of civil society actors. The question is to know if the professionalization of the combination may cause a change in the associative structure towards a system that can be compared to a private practice of similar.

These associations reach or not a certain degree of organization and structure considered of professional? 
Keys-words: Associative practices, Management skills, Isomorphism, Intention.

Este artículo se centra en las asociaciones profesionales, identificando formas de gestión de las competencias de los actores de la sociedad civil. El planteamiento es de si la profesionalización de la combinación puede causar un cambio en la estructura asociativa de un sistema que se puede comparar con una práctica similar de la organización privada.

¿Logran estas asociaciones alcanzar o no un cierto grado de organización y estructuración consideradas de nivel profesional?

Palabras claves: prácticas asociativas, capacidad de gestión, isomorfismo, cambio.

\section{Introduction}

La professionnalisation des associations est une problématique qui depuis plusieurs années se présente aux associations. Les réponses apportées par le monde de la recherche ou par les professionnels sont souvent partiellement efficaces, non pas au regard de la qualité des recommandations mais au regard de la nature particulière des mobiles qui conduisent les actions de ces associations, qui ne peuvent être subsumés sous le général.

En effet, chaque association possède son " esprit», et de cet esprit découle un mode comportemental spécifique qui conduit généralement les actions de chaque structure. Cette présence forte d'une véritable "personnalité organisationnelle» est totalement spécifique aux structures associatives et plus particulièrement à celles régies par la loi 1901.

L'objet de la recherche est la professionnalisation des associations par l'identification des compétences des acteurs associatifs. La question est de savoir si ces compétences ont pu être acquises au cours de la transformation de la structure associative vers une professionnalisation pouvant être rapprochée à une pratique privée 
d'organisation similaire, ou bien par une véritable pratique structurée d'outils de Gestion des Ressources Humaines. Pour ce faire, il a été pratiqué deux types de travaux. Les premiers travaux reposent sur la construction de systèmes de gestion de la formation professionnelle par les PME (SGFPC) sur la base de travaux de recherche menés en doctorat. Les deuxièmes travaux se composent de deux enquêtes à 10 ans d'intervalle sur les pratiques de gestion des compétences par les associations par l'intermédiaire de la FPC. La première enquête faite en 2001 par l'intermédiaire de questionnaires et d'interviews complémentaires en face en face a été complété par des données secondaires, recueillies en 2013 sur certaines des associations du premier échantillon par entretien et monographies de leur structure.

Nous verrons, dans un premier ce que l'on entend aujourd'hui par professionnalisation des associations (Chanut-Guieu, 2009). Pascal Ughetto \& Marie-Christine Combes (Ughetto P., Combes M.C., 2010) la résument assez justement : « la professionnalisation est cette organisation mise en face des problèmes productifs qui s'accroissent, en quantité et en complexité, quand les volumes atteignent des niveaux importants, que les promesses sur la qualité augmentent les exigences, et que cela conduit à s'interroger sur un « amateurisme» qui, jusqu'alors, n'apparaissait pas comme une difficulté.

\section{1. - Professionnalisation des associations}

Le questionnement principal qui a conduit notre recherche est le suivant: Les pratiques en matière de gestion des compétences des acteurs associatifs sont-elles sources de professionnalisation et de changement organisationnel?

La professionnalisation des associations est une question fondamentale en sciences de gestion. Elle passe par une acquisition de compétences organisationnelles. Mais ces compétences organisationnelles sont-elles spécifiques aux associations? Ces compétences organisationnelles ne peuvent se construire qu'avec l'émergence des compétences individuelles des acteurs qui composent la structure (Chanut-Guieu, 2009). Les associations étant par nature une structure particulière, composée à la fois de salariés et de bénévoles ${ }^{1}$, la question de leur développement par la professionnalisation prend tout

1. Par bénévole, nous traiterons des bénévoles opérationnels, étant donné que les dirigeants d'associations sont par nature bénévoles et donc dans l'obligation de se former face aux nouvelles obligations et responsabilités qui leur incombent. 
son sens aujourd'hui à travers l'étude des pratiques de formation professionnelle continue.

La professionnalisation est une problématique qui depuis plusieurs années se présente aux associations. Les réponses apportées par le monde de la recherche ou par les professionnels sont souvent partiellement efficaces, non pas au regard de la qualité des ces recommandations mais au regard de la nature particulière des mobiles qui conduisent les actions de ces associations, qui ne peuvent être subsumés sous le général.

En effet, chaque association possède son «esprit», et de cet esprit découle un mode comportemental spécifique qui conduit généralement les actions de chaque structure. Cette présence forte d'une véritable «personnalité organisationnelle» est totalement spécifique aux structures associatives et plus particulièrement à celles régies par la loi 1901.

Depuis 10 ans, les associations de forme 1901 doivent faire face à une mutation importante de leur environnement (Codello-Guijarro, 2012). La fiscalisation progressive de leurs activités qualifiées à tort ou raison d'économique, l'intensification des contrôles étatiques, génèrent une profonde transformation de leurs structures. Leur fonctionnement a souvent été synonyme dans l'esprit général de la simple forme égalitaire (Ughetto P., Combes M.C., 2010) :

ASSOCIATIONS $=$ BENEVOLES $=$ AMATEURISME

se transforme depuis plusieurs années, sous la pression environnementale et structurelle par la suivante :

ASSOCIATIONS $=$ RESPONSABILITES $=$ RECHERCHE DE COMPETENCES

Or face à cette évolution, qui s'éloigne de la recherche classique « d'implicateurs » du monde associatif néanmoins toujours présente, vers la recherche de gestionnaires ou de juristes, l'offre de formation de l'université française, rare dans les années 2000, commence à s'enrichir (Chanut-Guieu, 2009). Au phénomène de l'opposition éthique du gestionnaire et du bénévole (et encore plus du volontaire), l'association adapte sa stratégie pour survivre.

A ce besoin de compétences pointues, à la rareté absence de formation, s'ajoute le problème des acteurs des associations, qui, ayant "appris sur le terrain », désirent faire une pause dans leur parcours professionnel pour faire le point sur leur savoir-faire. Ce besoin de théorisation de leurs compétences se justifie par la nécessaire obtention de légitimité (Bernardeau, Moreau, Denis, 2008). Une organisation dans l'obligation conjoncturelle de se structurer, glisse-t-elle vers l'instrumentation pour se professionnaliser? 
Dans les secteurs particuliers de l'humanitaire ou de la recherche, nous constatons également qu'à la mutation environnementale législative se greffe une profonde modification du paysage associatif (Bidet, 2003). Ces organisations sont de plus en plus nombreuses sur ces deux secteurs et ainsi, les fonds doivent être «partagés » entre plus de structures. Un sentiment « concurrentiel» prend alors naissance (Borzaga, C. \& Defourny, J. Eds, 2001). A l'heure actuelle, pour obtenir des fonds, l'association doit justifier son activité. La justification entraînant la connaissance et la maîtrise du fonctionnement interne, l'organisation essaye alors de se structurer de manière à posséder un système d'information interne permettant de répondre à cette justification (Sousi G., 2011).

C'est ainsi qu'intervient un autre phénomène directement lié au précédent : la volonté d'indépendance financière vis à vis de fonds publics révisables, afin d'assurer la survie de l'association. La recherche active de financements propres par différentes voies devient une nécessité et de façon identique que l'on ne parlera pas de « concurrence», on fera de la « communication externe» plutôt que du « marketing » (Béji-Bécheur A., Diaz Pedregal V., 2008).

Deux hypothèses peuvent être posées :

Ces associations arrivent- elles à atteindre un certain degré d'organisation et de structuration jugé professionnel?

Quels rapprochements opérer avec le secteur économique et plus particulièrement avec celui des PME afin d'identifier un modèle de changement organisationnel?

Ughetto \& Combes (Ughetto P. \& Combes M.C., 2010) posent très bien les deux premiers sens au moins, du terme de «professionnalisation » qui s'oppose surtout à « amateur » (c'est-àdire à l'ignorance ou à l'empirisme de celui qui pratique l'activité de manière occasionnelle et sans préoccupation d'efficacité ou de rendement) et rime avec manque de méthode, de systématicité, de connaissance profonde des bases du métier. L'entreprise - peut-être, d'ailleurs, moins l'entreprise réelle que la représentation que l'on se fait de ce monde dans le cadre de l'univers associatif - est alors présente du point de vue de la capacité très puissante qui est la sienne de proposer, sans considération pour les spécificités associatives, des méthodes éprouvées. » 


\section{2. - Méthodologie et présentation des échantillons}

Tableau 1 : La structure des échantillons utilisés

\begin{tabular}{|r|r|r|r|r|}
\hline PME & salariés & $\begin{array}{l}\text { Associations } \\
\text { (enquête 2013) }\end{array}$ & Salariés & taille Bénévoles (effectif moyen) \\
\hline 10 & $>450$ & 2 & $>1000$ & $>1000$ \\
\hline 27 & $>350$ & & & 357 \\
\hline 32 & $>250$ & 8 & $>250$ & 90 \\
\hline 18 & $>150$ & 12 & $>150$ & 23 \\
\hline 22 & $>50$ & 16 & $>50$ & \\
\hline 109 & & 38 & \multicolumn{2}{r}{} \\
\cline { 2 - 4 } & & & &
\end{tabular}

\section{a. Les associations}

Deux enquêtes ont été menées à 10 ans d'intervalle pour les associations sur la base de questionnaires, d'entretiens semi-directifs et de monographies organisationnelles. Les résultats de la première enquête ont été retraités sur les 38 associations qui ont répondu à la deuxième enquête aux fins de disposer d'échantillons comparables dans le temps. Les associations retenues relèvent toutes du secteur des services (hors associations d'aide à domicile qui ont l'obligation d'employer des salariés formés ou de les former, d'associations de loisirs et d'éducation populaire, et d'associations sanitaires et sociales, seulement $10 \%$ de l'ensemble mais $45 \%$ du budget total des associations...) ainsi que les entreprises. Il s'agit exclusivement d'associations employeurs.

Rappelons que sur 1,3 million d'associations estimées vivantes, seules 183000 sont employeuses (RECMA, 2012), la question la professionnalisation des bénévoles et des salariés ne se posant pas tout à fait dans les mêmes termes dans le cas contraire.

Au total, nous avons renoué un contact effectif avec 38 associations de l'échantillon de base. Les autres structures ayant disparues, ou ne désirant plus participer à une étude organisationnelle.

\section{b. Les choix méthodologiques pour les travaux sur les PME}

Nos choix méthodologiques concernant l'approche du terrain pour les PME, le recueil des données et leur traitement sont les suivants.

Dans un premier temps, observatrice non participante pour un projet financé par la DFP PARIS, ensuite nous avons mené des entretiens face à face avec des experts, praticiens ou professionnels, pour parler de la Formation Professionnelle Continue. Nous avons 
conclu notre confrontation avec le terrain par deux actions distinctes : l'enquête par questionnaire et la vérification des principaux résultats empiriques.

Il s'agissait donc d'entretiens avec des experts, d'entretiens avec des responsables de formation, des directeurs des ressources humaines ou P.D.G., puis une enquête par questionnaire sur un échantillon plus large (raisons, présentation du questionnaire, mesures prises pour éviter les risques de biais ou d'erreurs...). Les deux premiers points relèvent d'une démarche exploratoire.

Les entretiens exploratoires ont été échelonnés dans le temps (2 ans). Ils nous ont permis de clarifier les variables et les composants d'une gestion de la formation professionnelle continue. Nous avons mené successivement six entretiens. Nous avons également utilisé deux méthodes exploratoires complémentaires: la pratique professionnelle en tant qu'enseignante en formation continue et la participation à un projet, sous convention de recherche, lancé par la délégation à la formation professionnelle de Paris (observation non participante).

L'étude empirique nous a permis de rassembler des données sur les concepts d'investissement, d'investissement immatériel en formation, de retour sur investissement et de création de valeur d'entreprise. L'enquête nous a renseigné également, après analyse des informations issues des observations sur, la validité ou l'invalidité, de nos hypothèses empiriques. Nous avons donc construit un guide pour les entretiens de test (6 P.M.E.). A partir de ce modèle, nous avons élaboré le questionnaire d'enquête (selon le même squelette).

Par la suite, pour valider les résultats obtenus, nous avons procédé à une enquête par questionnaire auprès de $153 \mathrm{PME}$, obtenant ainsi 44 questionnaires exploitables. Nous avons procédé à un dépouillement global de l'ensemble des questionnaires et entretiens afin de faire ressortir des groupes primaires de réponses à certaines questions, notamment à celles de l'investissement, l'investissement formation et du retour sur investissement. Ces groupes primaires ou catégories de réponses ont été affinés, validés ou invalidés lors de l'analyse des données.

Dans un second temps, nous avons vérifié la structure organisationnelle de chaque entreprise, à savoir la confirmation téléphonique, ou entretiens sur place pour les monographies d'entreprises, des postes et personnes titulaires, des rôles réels au niveau de la gestion stratégique des actions. Dans certains questionnaires, le directeur des ressources humaines n'apparaît pas dans le processus de gestion de la formation. Nous avons demandé confirmation de l'absence fonctionnelle et physique de cet acteur dans 
l'entreprise (entretien téléphonique ou plus rarement par courrier). Il est apparu qu'en l'absence fonctionnelle du directeur des ressources humaines, la fonction est assurée par la direction générale et notamment le président directeur général.

En revanche, les acteurs nommés "responsables hiérarchiques » directs (agents de maîtrise et encadrement intermédiaire) ne sont pas mentionnés dans le processus de gestion de la formation par les P.M.E., ce n'était pas du fait de l'absence fonctionnelle ou physique de tels acteurs, mais bien une absence de participation dans ce système de gestion. Dernière précaution prise qui concerne les catégories de personnel formées. A cette question, il est proposé plusieurs catégories de personnel dont les ouvriers non qualifiés. Parmi les réponses obtenues, certaines entreprises ne mentionnaient pas cette catégorie de salariés comme étant susceptibles d'être formée. Après vérification auprès de ces P.M.E., il apparaît que cette catégorie de personnel est absente de l'organisation des entreprises.

La principale méthode d'analyse des données empiriques relève de l'analyse qualitative. La mesure des relations entre les variables conformément aux hypothèses ou la comparaison de relations observées aux relations théoriques attendues par hypothèse, la mesure de l'écart entre les deux est fait au moyen de l'analyse de contenu en utilisant des outils comme des matrices, diagrammes, graphes... Largement inspirée des propos de Faule (Faule, 1982) et de Jick (Jick, 1979) pour qui, combiner la recherche qualitative et quantitative ne fait qu'amplifier la validité des résultats. La démarche de base fut de collecter les données par questionnaire puis par entretiens semi directifs. Cette méthodologie «tandem » permet l'accès à des niveaux différents de la réalité (Bryman, 1989).

En ce qui concerne les différences matrices construites pour l'analyse des comportements, des systèmes, puis des profils d'entreprise, nous nous sommes aidée des travaux de Miles et Huberman (1984) qui ont élaboré des techniques spécifiques pour analyser des données qualitatives. Leur idée présente une variété de possibilités, allant des graphes de simulation ou d'observations des données présentes, sans pour autant détruire le sens des données. Une partie de l'exploitation des questionnaires d'entreprises nous a demandé de mettre sous forme d'équations, un certain nombre de données.

Nous avons déterminé pour chaque niveau d'analyse, un certain nombre de variables explicatives qui ont été étudiées et précisées lors de chaque étape. La démarche de construction empirique peut être présentée comme suit : 
Nous allons maintenant présenter les systèmes de gestion de la formation professionnelle continue qui ont été identifiés lors de cette première recherche.

\section{3. - Les systèmes de gestion de FPC identifiés dans les PME}

Nous avons procédé de la même manière pour les PME. Nous avions obtenu quatre systèmes primaires de gestion de la formation : Le système participatif, le système parcellaire, le système centralisateur, le système participatif restreint. Pour chacun de ces systèmes, nous avons utilisé des critères de classification qui sont :

\section{a) Le système participatif}

Le système participatif est un système de gestion de la formation où l'ensemble des tâches ou étapes de la décision est décentralisée au maximum. Chaque acteur participe à un degré différent ou identique au processus de gestion de la formation, de façon plus ou moins forte.

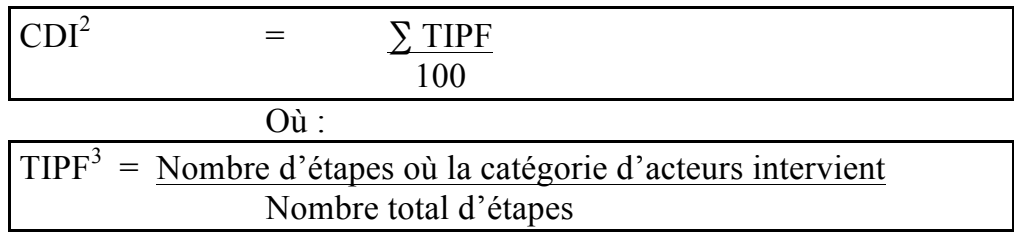

Condition d'existence du système : CDI $\geq 200 \%$.

\section{b) Le système parcellaire :}

Le système parcellaire recouvre une gestion fractionnée (par petits morceaux) de la formation mais surtout, chaque phase est gérée indépendamment et par des acteurs différents. La gestion est éclatée au sein des différentes catégories d'acteurs.

Condition d'existence du système : CDI $\leq 100 \%$.

\section{c) Le système centralisateur :}

Le système centralisateur illustre une gestion où une seule catégorie d'acteurs détient au moins $80 \%$ des actions de formation ou intervient dans au moins $80 \%$ voir $100 \%$ des étapes de la gestion de la formation

2. Coefficient d'Intensité d'Intervention.

3. Taux Individuel de Participation Fonctionnelle. 
Conditions d'existence du système : Un acteur ayant un TIPF égal à 100 ou supérieur ou égal à $80 \%$, avec au moins tous les autres acteurs possédant un TIPF inférieur ou égal à $60 \%$.

\section{d) Le système participatif restreint:}

Le système participatif restreint est un système de gestion de la formation où deux catégories au moins d'acteurs se partagent à part égale plusieurs étapes de la gestion de la formation.

Condition d'existence du système: Il existe un partage équiprobable des actions de la gestion de la formation entre u moins deux catégories d'acteurs. Exemple : Direction générale et Hiérarchie indirecte ou directe à $80 / 80,40 / 40, \ldots$

En croisant les systèmes de gestion et les outils, nous établissons des comportements d'entreprises vis à vis de la formation, reflet des pratiques de gestion. Les résultats primaires ont donné cinq comportements pour huit entreprises. Ces comportements sont :

1/ Le comportement d'investisseur - participatif ;

2/ Le comportement d'investisseur - participatif restreint

3/ Le comportement d'investisseur - centralisateur ;

4/ Le comportement budgétaire - parcellaire ;

5/ Le comportement budgétaire - participatif restreint.

Nous avons croisé les quatre systèmes de gestion avec les trois groupes d'outils, pour obtenir les différents comportements d'entreprises sur une population de 40 P.M.E.. Le comportement le plus fréquent sur cette population est un comportement mono - outil à court terme avec un système participatif restreint. Il s'agit du comportement primaire nommé «budgétaire - participatif restreint ». Nous remarquons que pour 40 entreprises, nous obtenons seulement deux comportements supplémentaires, à savoir: un comportement budgétaire - centralisateur et un comportement d'investisseur parcellaire.

Ce qui, proportionnellement, pour le nombre d'entreprises complémentaires (40 P.M.E.) par rapport au nombre primaire d'entreprises (8 monographies), laisse à penser que le nombre de comportements est limité malgré le nombre important de combinaisons possibles entre les systèmes de gestion (4) et les groupes d'outils (3), soit 12 possibilités. Toutes choses égales par ailleurs, nous complétons (en gras) les résultats primaires sur les comportements :

$\rightarrow$ Tout système de gestion de type centralisateur ou participatif est accompagné d'un comportement d'investisseur sauf pour les systèmes centralisateur avec un CDI inférieur à 180 . 
$\rightarrow$ Tout système de gestion de type participatif restreint est accompagné d'un comportement court terme en l'absence de GPEC et d'un comportement d'investisseur à moyen terme dans le cas contraire.

Sur une population plus étendue de P.M.E., nous infirmons le résultat primaire selon lequel à un certain niveau de CDI, les entreprises à systèmes participatif restreint ou parcellaire opèrent une gestion à long terme. Il paraît également infirmé le fait que plus l'entreprise se situe dans le haut de la matrice, plus elle gère la formation à long terme.

Nous pensons, qu'indépendamment de sa position dans la matrice, l'entreprise peut avoir un comportement à long terme, ce qui ne signifie pas d'ailleurs qu'il soit qualifiable d'investisseur.

Nous remarquons également que pour l'axe central primaire «Coût-Investissement» ne peut plus s'appliquer globalement à la matrice des systèmes. En revanche l'analyse précédente fait ressortir que plus le CDI augmente dans chaque catégorie, plus le comportement en matière d'outils se situe sur le long terme, à savoir :

Schéma 3 : Matrice de positionnement des structures

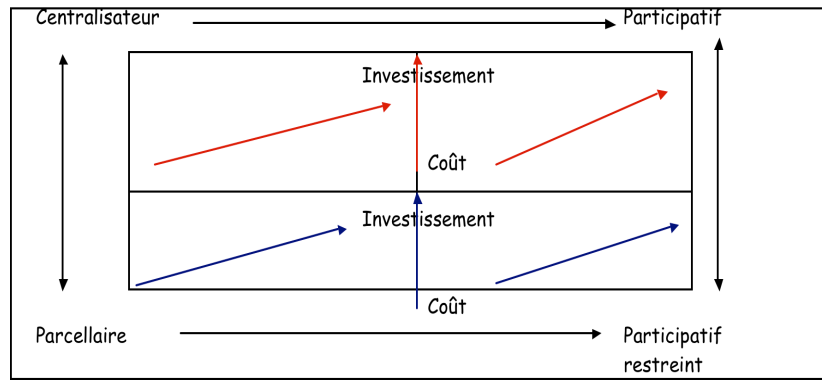

et non plus la forme :



Les différents discours primaires identifiés sont le discours démagogique, le discours social, le discours du stratège prudent et le discours de l'économico - stratège. 
Les variables utilisées pour la construction des discours sont :

1/ La valeur supplémentaire apportée par des ressources humaines formées ;

2/ L'égalité «toute dépense de formation est un investissement $\gg$;

3/ La définition de la nature de la formation;

4/ Les attitudes face à la formation.

Les différents discours primaires ont la structure suivante:

Schéma 4 : Structures des discours primaires

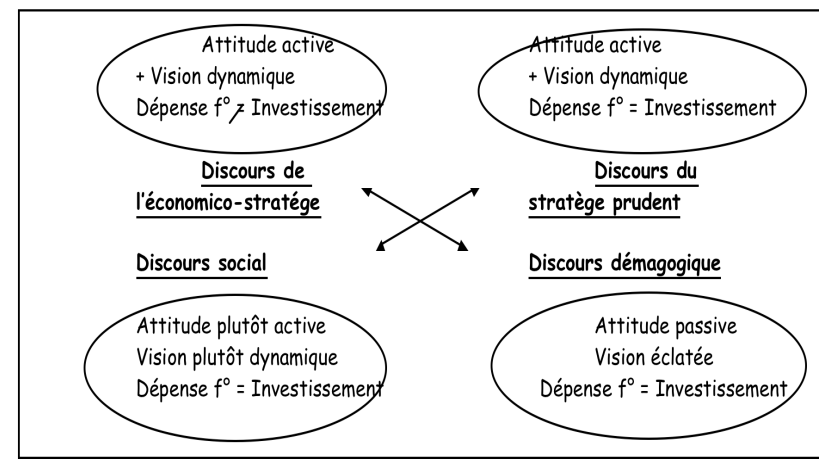

Après analyse des 40 réponses, toutes les entreprises ont pu être classées selon les mêmes critères de définition des discours et dans les types définis au stade primaire.

Nous rappelons que les critères utilisés sont notamment :

Schéma 5 : Les critères des attitudes et visions utilisés

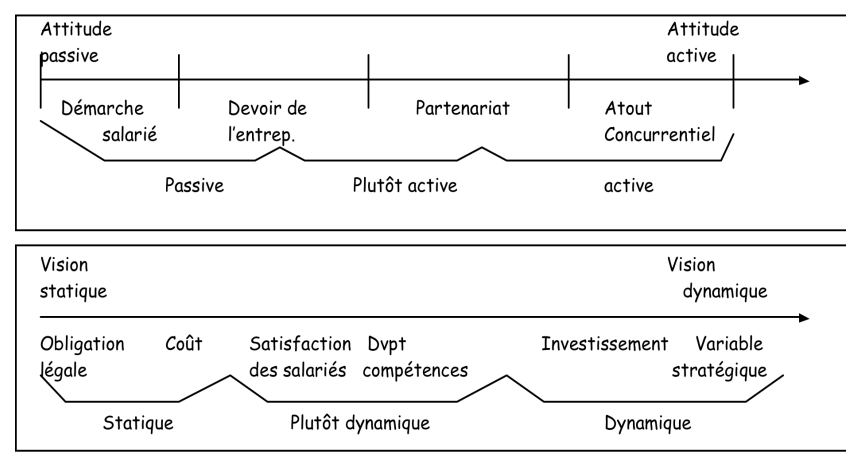

Nous constatons que les deux discours majoritaires sont les discours du stratège prudent et social. Le discours de l'« économico-stratège » 
représente $17,50 \%$ de la population, alors que pour chaque discours majoritaire, la proportion est de 37,5\% chacun, soit $75 \%$ de la population. Aucune entreprise n'a tenu de discours fondamentalement différent de ces discours primaires. Nous pouvons donc conserver ces résultats. Si nous reprenons les définitions primaires des familles, nous avons :

Famille 1: Discours de l'économico stratège ou stratège prudent associé à un comportement d'investisseur soit centralisateur, soit participatif ;

Famille 2: Discours social associé à un comportement budgétaire participatif restreint quel qu'il soit ;

Famille 3: Discours social associé à un comportement d'investisseur participatif restreint;

Famille 4: Discours démagogique associé à un comportement budgétaire parcellaire ;

Famille 5: Discours du stratège prudent accompagné d'un comportement budgétaire participatif restreint.

Lors de l'analyse antérieure des 40 entreprises, nous avons obtenu deux comportements complémentaires: un comportement budgétaire (centralisateur) et un comportement investisseur (parcellaire).

L'axe des comportements a changé pour comprendre désormais sept comportements au lieu de cinq. En scindant le plan selon les mêmes règles, en quatre zones, nous repérons les familles primaires. Si nous élargissons le champ de ces familles primaires aux nouveaux comportements limitrophes, nous intégrons le discours d'investisseur parcellaire aux familles F3 et F1, pour obtenir les familles F3' et F1'. De façon similaire, nous raccrochons le discours budgétaire centralisateur au budgétaire participatif restreint, pour obtenir les familles F5' et F2'.

\section{Constats :}

1/ La famille F4 ne concerne aucune entreprise de notre population nouvelle.

2/ La famille F5 concerne une seule entreprise.

3/ Les profils d'entreprises les plus fréquents se situent en F1' et F3'.

En synthèse, toutes choses égales par ailleurs, nous pouvons confirmer certains résultats primaires.

$\rightarrow$ Lorsque les entreprises ont un discours d'économico stratège ou de stratège prudent, leur comportement relève majoritairement d'un comportement d'investisseur (19 cas sur les 21 cas ayant un discours stratégique). De plus, le discours d'économico stratège relève exclusivement d'un comportement d'investisseur. 
$\rightarrow$ Le discours social est généralement associé à un comportement d'investisseur (10 cas sur 15$)$, et plus particulièrement à celui d'investisseur participatif restreint (4 cas sur 15).

Nous confirmons également que le discours de stratège prudent est associé indifféremment des comportements d'investisseur. Nous obtenons des comportements budgétaires plutôt avec des discours sociaux.

En conclusion, nous pouvons avancer, dans le cadre de cette recherche, que lorsque les entreprises ont un discours stratégique de la formation, elles se situent dans des comportements plutôt d'investisseurs. L'évolution apparemment de la démarche de ces P.M.E. en matière de gestion de la formation, doit être relativisée au regard de deux phénomènes principaux :

- La crise économique force les entreprises, mêmes les plus petites, à étudier et gérer les dépenses quelles qu'elles soient.

- Le phénomène de la certification des P.M.E. en qualité (normes ISO), oblige ces mêmes petites structures à posséder un plan de formation annuel, pour remplir le cahier des charges de la qualité.

De plus, le biais inhérent au terrain de recherche : Le fait que les P.M.E. répondent aux questionnaires présuppose une préoccupation particulière des problèmes de gestion de la formation professionnelle. Sachant que nous avons obtenu un taux de réponse de $14,30 \%$ à la première enquête (questions ouvertes et fermées, longueur : 6 pages), et de 26,80\% à l'enquête de validité (questions fermées, longueur : 2 pages), nous estimons réelle la préoccupation des P.M.E. en matière de formation.

\section{4. - Les résultats des enquêtes pour les associations}

L'analyse des données recueillies nous permet de constater que dans $70 \%$ des cas, la structuration de l'association passe par la fonctionnalisation. En effet, $48 \%$ des associations (38 au total) possèdent désormais une direction des ressources humaines. $\mathrm{La}$ quasi - totalité (98\%) travaille avec des bénévoles opérationnels. A ces deux constats, il nous faut préciser certains points. A propos du premier constat :

Les $48 \%$ d'associations possédant une direction des ressources humaines sont des structures dont l'effectif salarié se situe à plus de 49. Le même phénomène est d'ailleurs observable dans les PME mais avec un seuil de 200 salariés. De façon parallèle, ce sont les Grandes Associations (GA) qui fonctionnent le plus souvent avec le plus grand 
nombre de bénévoles opérationnels (jusqu'à 1000 bénévoles dans notre population).

Un deuxième constat nous paraît intéressant. Pour $89 \%$ de ces associations, la formation est un outil de développement des compétences et un investissement.

$56 \%$ d'entre elles considèrent la formation comme une variable stratégique de l'organisation. La formation est l'un des premiers maillons à se mettre en place lors d'une évolution structurelle et environnementale. En effet, souvent à la recherche d'identification et de mise en œuvre de compétences nouvelles et adaptées aux nouveaux facteurs, la formation est souvent considérée comme le moyen idéal pour résoudre le dysfonctionnement.

Rappelons que les résultats de la recherche sur les PME, nous a amené à faire certains constats. Le discours des PME sur la formation comme un investissement se trouve majoritairement associé à l'absence d'outils de gestion et de management des compétences (Plan de formation, Gestion Prévisionnelle des Emplois et des Compétences...) d'une portée prévisionnelle supérieure ou égale à un an. Ce qui reflète un discours d'investisseur souvent décalé avec un système de gestion parcellaire ou centralisateur à faible horizon temporel.

De même que les réponses obtenues concernant le concept d'investissement formation par les associations comme un partenariat salarié / organisation (67\%), et un devoir de l'organisation vis à vis de son salarié (45\%) pouvant apparaître comme « anormalement social» pour une entreprise, mais "naturel » pour l'association. 100\% des associations avance une reconnaissance de la formation professionnelle dans l'organisation. En revanche entre promotion $d u$ salarié (28\%) et gratification salariale (20\%) les avis restent partagés. A l'identique des PME, les associations (49\%) affirment que former les ressources humaines apporte une valeur supplémentaire à l'organisation et que cette valeur est de nature stratégique (Amélioration $\mathrm{du}$ climat social, Qualification, Motivation du personnel...). Sur ce point encore, nous retrouvons ce désir de rester "social» tout en voulant répondre à la pression de la mutation des facteurs externes de l'association. Très rarement, ces associations possèdent une GPEC (10\%) pour leurs salariés, et encore moins pour leurs bénévoles opérationnels $(8 \%)$.

Toutes choses égales par ailleurs, ceci rejoint notre constat précédent. A savoir qu'un discours d'investisseur en termes de formation, associée à un début de structuration organisationnelle, est souvent corroboré à une absence d'outils de gestion à moyen ou long terme. A ce propos, l'entretien annuel d'appréciation est pratiqué 
pour la moitié seulement de nos associations, auprès de leurs salariés, et quasiment absent $(0.5 \%)$ pour les bénévoles opérationnels. Nous pouvons dire que le bilan de compétences est absent $(0.28 \%)$. En ce qui concerne les variables actuelles de pilotage de la formation mises en place par les organisations, $78 \%$ d'entre elles possèdent un plan de formation inférieur à un an, et $62 \%$ un plan de formation inférieur ou égal à 3 ans. En ce qui concerne l'affectation des dépenses de formation, nous constatons que les bénévoles opérationnels bénéficient à parts égales des formations avec les techniciens et agents de maîtrise. De façon identique aux PME, les ingénieurs et cadres $n$ 'en bénéficient que dans $25 \%$ des cas. Ce qui représente pour les associations l'effort de formation suivant (en \% de la masse salariale).

Figure 1 : Effort de formation des associations de l'échantillon en 2012


Remarque : Le pourcentage des dépenses de formation consacré aux bénévoles est inférieur ou égal à $1 \%$.

\section{5. - Discussion et conclusion}

A ce stade de l'analyse, nous pouvons essayer de répondre à notre interrogation de départ.

Un premier constat : face aux mutations environnementales, l'association, qu'elle soit petite, moyenne ou grande opère une première structuration organisationnelle par une instrumentation primaire de sa gestion des compétences des permanents (salariés), puis, par effet de "glissement" qui s'achemine vers une instrumentation secondaire en direction des bénévoles. Nous pourrions expliquer l'évolution organisationnelle de l'association par quatre phases successives :

- La création de l'association

- Le développement de l'association

- La structuration des Ressources Humaines

- Le déclin de l'association. 
L'association est en zone de fragilité à partir du stade de son développement si elle ne prend pas en considération des besoins de structuration de ses ressources humaines, tant salariées que bénévoles. Il est alors intéressant de remarquer que le degré de la zone de fragilité est directement lié à la volonté politique de développement de l'association.

En effet, l'association peut prendre la direction d'une structuration par l'instrumentation avec une recherche nette d'indépendance financière afin d'assurer la pérennité de l'organisation. Ou alors, dans un second cas, choisir l'instrumentation partielle ou nulle, et se diriger vers une phase de déclin, sauf en cas de recherche systématique d'indépendance financière. Mais, nous précisons qu'une telle action ne peut s'opérer que dans un cadre organisé. Ces propos sont à nuancer car l'instrumentation se fait d'une part sous la pression structurelle (évolution de l'association en taille) mais également sous la pression environnementale (renforcement des contrôles, justification des besoins...). Dans cette perspective d'évolution, l'association est dans l'obligation de connaître son existant, et plus que partout ailleurs, l'essence de l'organisation repose ici sur l'homme, qu'il soit bénévole ou permanent. La recherche de compétences se voit confirmée notamment par l'explosion de la demande de formation des associations pour leurs salariés et bénévoles.

Sur notre interrogation concernant l'isomorphisme des pratiques associatives en matière de formation professionnelle continue, il semblerait apparaître une différence de positionnement au regard des discours et des pratiques identifiés. En effet, la majorité des associations se sont positionnées sur des systèmes de gestion de la formation à discours social. Seule la famille F3' semble être commune aux pratiques de gestion de la formation pour ces deux types de structures, à savoir un discours social accompagné d'un comportement d'investissement quel qu'il soit. 
Tableau 2 : répartition et comparaison PME/Associations

\begin{tabular}{|l|l|l|l|l|}
\hline Familles $^{4}$ & $\begin{array}{l}\text { Effectif } \\
\text { PME }\end{array}$ & Proportion & $\begin{array}{l}\text { Effectif } \\
\text { Associations }\end{array}$ & Proportion \\
\hline F1' & 14 & $35 \%$ & 3 & $7.9 \%$ \\
\hline F2' & 10 & $25 \%$ & 14 & $36.8 \%$ \\
\hline F3' & 12 & $30 \%$ & 12 & $31.6 \%$ \\
\hline F4' & 3 & $7.5 \%$ & 1 & $2.6 \%$ \\
\hline F5' & 1 & $2.5 \%$ & 8 & $21.1 \%$ \\
\hline
\end{tabular}

En revanche, les associations associent aux mêmes discours, les mêmes pratiques aboutissant aux mêmes systèmes de gestion participatif, participatif restreint, centralisateur et parcellaire.

L'isomorphisme associatif semble donc être modéré au regard du discours social et du comportement d'investisseur sous contrainte budgétaire des associations. Ce caractère social de l'investissement formation est ressorti des propos recueillis auprès des associations comme lié directement au caractère spécifique de l'association.

Propos d'un directeur d'association: «Ici, on fait en sorte que tous soient formés, bénévoles et salariés. Il est plus qu'important aujourd'hui qu'une association puisse disposer de personnes qualifiées. Nous avons tellement de contraintes et un fonctionnement qui se complexifie de jour en jour, qu'en fait, ce qui nous différencie encore aujourd'hui des entreprises, c'est que les personnes qui travaillent chez nous ont un but différent et recherchent quelque chose de différent. Ça c'est l'esprit associatif ».

Enfin, toutes choses égales par ailleurs, les pratiques de Gestion des Compétences des Grandes Associations aident à leur professionnalisation par un changement organisationnel sur les points suivants :

Le premier point concerne la mise en place progressive d'outils d'appréciation du personnel (entretien individuel, GPEC...) et de gestion de la formation (Plan de formation et durée des plans,...) en lien direct avec le degré d'évolution organisation et structurel (taille) de l'association.

4. Famille F1' : Discours de l'économico stratège ou de stratège prudent accompagné d'un comportement d'investisseur quel qu'il soit / Famille F2' : Discours social, accompagné d'un comportement budgétaire quel qu'il soit / Famille F3' : Discours social accompagné d'un comportement d'investisseur quel qu'il soit / Famille 4': Discours démagogique accompagné d'un comportement budgétaire ou d'investisseur / Famille 5' : Discours du stratège prudent accompagné d'un comportement budgétaire. 
Le deuxième point est relatif à la structuration organisationnelle par l'apparition d'une nette tendance fonctionnelle des ressources humaines à un seuil de 49 salariés, contre 200 pour les PME. Un rapprochement peut être opéré dans la difficulté rencontrée par certains salariés pour accéder à la formation.

La divergence fondamentale réside essentiellement dans une gestion des bénévoles qui reste en décalage avec une gestion des compétences tournée quasi exclusivement vers les salariés.

A ce niveau, il nous faut différencier les volontaires à l'expatriation et bénéficiaires d'un suivi spécifique, d'une gestion de la compétence du départ et de la réinsertion au retour.

En revanche, toutes choses égales par ailleurs, pour les bénévoles, nous restons le plus souvent face à une gestion parcellaire tournée vers un aspect quantitatif.

Etudier le comportement des associations en matière de gestion des compétences relève d'un questionnement actuel. Devons - nous, comme tenté depuis plusieurs années aborder l'étude du mouvement de structuration des associations et de leur professionnalisation sous l'angle d'une éventuelle transférabilité des méthodes de gouvernance des entreprises, ou alors se positionner comme concepteur de méthodes nouvelles de gouvernances dédiées à ces structures?

L'isomorphisme instrumental (Bidet E., 2003) est-il alors une condition de survie de ces structures au détriment du développement et de la conservation de l'esprit et de la personnalité de ces organisations subsumées alors sous l'isomorphisme managérial?

Des modèles classiques de gouvernance des entreprises, nous pouvons retenir que l'élément fondamental qui différencie l'entreprise de l'entreprise, mais dont la frontière tend à s'effacer petit à petit et selon les structures, est «l'esprit» de l'association. Tout l'enjeu de cette approche réside dans le fait que s'intéresser alors au mobile qui conduit l'action de l'organisation serait une possibilité de différenciation des pratiques entreprises et associatives. Encore faut que sous le concept associatif, et ce qui n'est pas le cas, nous ne trouvions que des associations fonctionnant «comme des associations ». Le problème ne semble pas alors se trouver dans la nature même de la structure mais plutôt dans son mobile.

En conclusion de ce propos et pour bâtir une première étape à cette recherche, nous pouvons avancer que l'évolution des associations suit un phénomène quasi parallèle à celui des PME tant au niveau des seuils de taille ou même des dysfonctionnements en matière de gestion des ressources humaines, mais qu'il faut veiller aux risques que peut engendre ce type d'isomorphisme (Bidet E., 2003), 
bien qu'il soit une possibilité de mener à la professionnalisation des associations.

Ces premiers résultats nous confirment le fait de l'utilité d'une recherche basée sur des pratiques de gestion des compétences par une lecture de pratiques spécifiques du secteur associatif afin de les accompagner dans leur évolution.

La piste de recherche essentielle issue de cette étude nous amène à une réflexion plus large concernant les modèles de lecture de ces pratiques et de l'évolution de ces pratiques en dehors de toute nature de l'organisation. En effet, il nous faut réfléchir à une lecture selon la nature du mobile qui conduit l'action de l'organisation comme point de départ en identifiant les sources réelles des motivations de l'organisation à agir pour le développement des compétences. Une analyse plus poussée sur ces mobiles pourrait faire apparaître des similitudes quand aux pratiques organisationnelles au regard des mobiles, indépendamment de la nature de l'organisation. Ainsi le concept de "personnalité organisationnelle» ou «esprit» pourrait être la voie d'identification et de fondement d'une lecture adéquate de pratiques spécifiques subsumées sous le concept de l'intention ou du mobile qui conduit l'action organisationnelle.

\section{RÉFÉRENCES BIBLIOGRAPHIQUES}

Bési-Bécheur A., Diaz Pedregal V. \& Ozcaglar-Toulouse (2008), Fair trade - just how 'fair' are the exchange? Journal of Macromarketing, 28, 1, p. 44-62.

BIDET E. (2003), «L'insoutenable grand écart de l'économie sociale: isomorphisme institutionnel et économie solidaire », Revue du MAUSS, ${ }^{\circ}$ 21, p. 162-178.

Borzaga C. \& Defourny J. (Eds) (2001). The emergence of social enterprise. London, UK : Routledger. Nyssens, (2006).

Chanut-Guieu C., «La professionnalisation de la fonction de bénévole: quand l'Etat impulse le changement », Management \& Avenir 7, 27, p. 1330

BRYMAn A., Research Methods and Organization Studies, Routledge, 1989.

Codello-Guijarro P. (2012), Professionnalisation dans le monde associatif: Comment donner une place à toutes les parties prenantes de l'association?, Editions Universitaires Européennes.

FAULE G., « The study of network structuring in organizations trough the use of methods triangulation », Western Journal of Speech, 1982

JICK T., Mixing qualitative and quantitative methods: triangulation in action, Cornell University, 1979. 
KAnt E. (écrit en 1781, 2003), Critique de la raison pure, Essai Folio Gallimard.

FranÇOIS, Y. (1998), Gestion Tridmensionnelle de la Formation Profesionnelle Continue : analyse et identification des discours, Thèse de doctorat en sciences de Gestion, IAE, université Jean Moulin Lyon 3.

Figures et tableaux :

Figure 1: Effort de formation des associations de l'échantillon en 2012

Tableau $1:$ Structure des échantillons

Tableau 2 : Répartition et comparaison PME/Associations

\section{Schémas :}

Schéma 1: Démarche de construction des systèmes de gestion de la FPC

Schéma 2 : Les niveaux d'analyse de la recherche sur les PME

Schéma 3 : Matrice de positionnement des structures

Schéma 4 : Structures des discours primaires

Schéma 5 : Les critères des attitudes et visions utilisés 